\title{
dibujo automático de planos complejos: control de posiciones ocupadas
}

JESUS ORTIZ HERRERA,

Dr. Ingeniero de Caminos.

Jefe del Departamento de Programación y Cálculo electrónico de MART-2 Gabinete Técnico, S. A.

$792-1$

sinopsis

\begin{abstract}
El diseño computarizado de estructuras de edificación a un nivel de automatismo prácticamente del cien por cien, exige la resolución de múltiples problemas de variada índole. De entre ellos, el autor selecciona un tema de especial interés, por su aplicabilidad genérica y por apartarse de los temas ya clásicos en el dimensionamiento automático de estructuras.
\end{abstract}

\section{INDICE}

1. Introducción.-2..$^{\circ}$ Delimitación de una etiqueta móvil.-3. ${ }^{\circ}$ Memoria de posiciones ocupadas. - 4. ${ }^{\circ}$ Criterio de ocupación.-5. Posicionamiento de una etiqueta.- $6 .^{\circ}$ Teoría del control ocupacional discreto en una dimensión.-7..$^{\circ}$ Ejemplo práctico.—8. ${ }^{\circ}$ Notación.

\section{INTRODUCCION}

El tema del diseño automático de estructuras es lo suficientemente atractivo como para haber suscitado (y continuar haciéndolo) gran cantidad de publicaciones e investigación a nivel mundial. El autor de estas líneas ha trabajado durante más de un año en la creación de un sistema de programas de ordenador para la "realización» de proyectos completos de estructuras mixtas (hormigón-acero estructural) de edificación; entendiendo por "realización», no sólo el cálculo estructural, cálculo de secciones, dimensionamiento óptimo, medición, etc., sino también la producción de memorias y planos, el almacenamiento (en disco magnético) de proyectos realizados, estadística de los mismos, etc. Usualmente, al hablar de diseño computarizado de estructuras o proyectos en general, se hace referencia a problemas de tipo matemático: optimización lineal o no lineal, teoría de grafos, etc., herramientas de indudable utilidad en el desarrollo de los cálculos; pero el trabajo necesario para su instrumentación práctica equivale realmente tan sólo a una pequeña parte de la ardua labor de normalización (de datos, criterios «locales» de cálculo, simbolismos, etc.), de ensamblamiento (de múltiples elementos de programación en intensa conectividad mutua) y de chequeo (seguridad razonable de ausencia de errores en la programación, en bancos de datos y tablas, etc.) que la puesta a punto de un tal sistema de programas requiere, en el cual toda eventualidad tiene que estar prevista: en el cálculo electrónico no hay lugar, por ahora, a la fantástica capacidad de improvisación y adaptación del cerebro humano.

Contémplese, por ejemplo, el estúpido problema siguiente (estúpido para un delineante «humano»): dado un plano a medio realizar, dónde colocar un nuevo grupo de símbolos, en una zona conflictiva del plano (si la zona es altamente conflictiva, el problema puede real- 
mente dejar de ser estúpido). Al delineante experimentado le bastará una inspección visual y un rápido proceso de integración mental apenas consciente, para seleccionar una posición óptima o, al menos, correcta; la máquina puede quizá realizar lo mismo mejor y en menos tiempo, pero tras una deliberación interna absolutamente "torpe», aunque increíblemente rápida.

La resolución automática del problema citado no es más que uno de los muchos subproductos teóricos de la labor comentada en el primer párrafo. Pero tiene el atractivo de constituir un algoritmo genérico, aplicable de modo bastante general para el dibujo computarizado de planos complejos (o para determinadas zonas conflictivas de los mismos), en los que se sucedan gran cantidad de elementos (no superponibles entre sí) de forma prácticamente aleatoria, lo cual invalida sistemas de acotaciones apriorísticas válidas para otros planos más simples. La solución elaborada por el autor para este problema, es el objeto de la presente exposición.

\section{DELIMITACION DE UNA ETIQUETA MOVIL}

Por «etiqueta móvil» (o simplemente, «etiqueta») entenderemos aquí un conjunto de símbolos de cualquier naturaleza, a dibujar con tamaño y posición relativa fijos, pudiendo sin embargo trasladarse y girar solidariamente. Se trata, por lo tanto, de elementos arbitrarios del dibujo, pero con posición no absolutamente predeterminada: la misma tiene que ser lo más próxima posible a una posición óptima, pero al mismo tiempo no debe interactuar con las etiquetas previamente dibujadas.

Supondremos que el criterio de interacción de cada etiqueta con las restantes se aplica sólo con las de aparición previa a la misma, ignorando las futuras (todavía indeterminadas). Esto tiene la ventaja de poder resolver uno a uno el posicionamiento de cada etiqueta; y conduce indudablemente a una solución correcta, aunque no necesariamente a la solución óptima (1), la cual debería obtenerse considerando globalmente todas las etiquetas en conflicto.

El primer problema es el de delimitar cada etiqueta; es decir, el de definir una cierta zona del plano asignable a la misma, e incompatible (al menos, dentro de ciertos márgenes) con las del resto de las etiquetas. Como no nos interesa el contenido del dibujo, en lo sucesivo denominaremos simplemente «etiqueta» a la zona del plano asignada a la misma.

Un sistema sencillo para delimitar cada etiqueta es identificarla con un conjunto

$$
E=\left\{r_{i} / 1 \leqslant i \leqslant n\right\}
$$

de cuadrados elementales $r_{i}$ («recuadros-móviles»), tal como se indica en la figura 1. Eligiendo el lado $(u)$ de cada recuadro con dimensión suficientemente pequeña, pueden siempre aproximarse etiqueta y conjunto de recuadros con precisión suficiente (en lo sucesivo llamaremos también «etiqueta» al correspondiente conjunto de recuadros $\left\{r_{i}\right\}$ ). Naturalmente, la elección de $u$ debe ser un compromiso entre imprecisión $(\propto u)$ y memoria necesaria $\left(\propto 1 / u^{2}\right)$.

Al ser cada elemento de etiqueta un cuadrado, su situación se define con un número reducido de parámetros: concretamente, basta dar un vector $\bar{V}$ de cuatro elementos (2), o «vector posición» del recuadro:

$$
\bar{V}=\left[\begin{array}{l}
x_{0} \\
y_{0} \\
\alpha \\
u
\end{array}\right]
$$

(1) Optima en el sentido de minimizar una cierta medida o "norma" de la separación entre el conjunto de posiciones de las etiquetas, y el conjunto de sus respectivas posiciones fijadas a priori como más deseables.

(2) Por su mucho uso, conviene añadir al vector $\overline{\mathrm{V}}$ dos términos más: $u \cos \alpha$ y $u$ sen $\alpha$, lo cual ahorra un número considerable de repeticiones del cálculo de $\cos \alpha$ y $\operatorname{sen} \alpha$. 


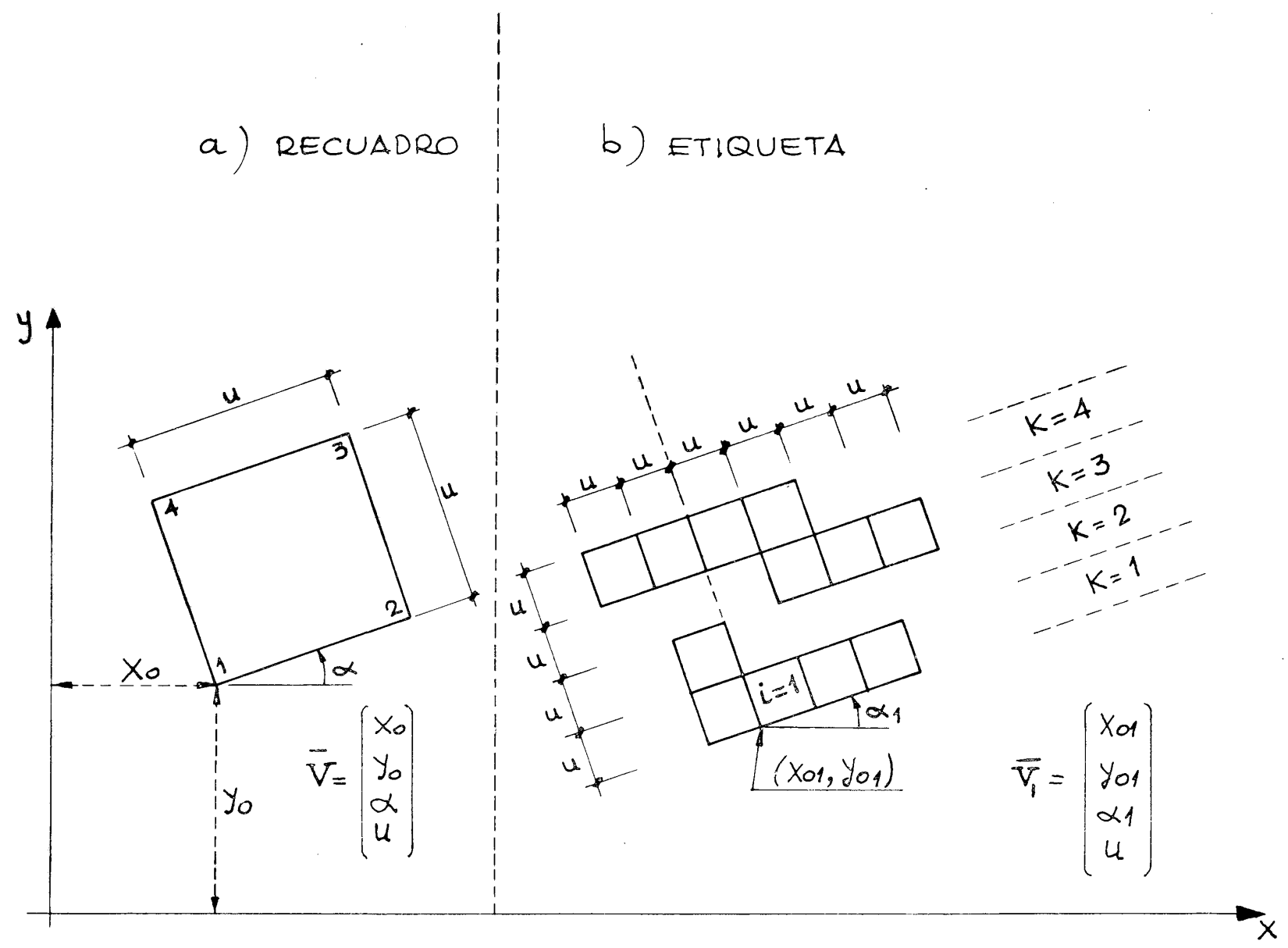

Fig. 1. Delimitación de etiquetas.

Las magnitudes $x_{0}, y_{0}$ (coordenadas de un vértice) y $\alpha$ (orientación) se indican esquemáticamente en la propia figura 1. Dadas $x_{0}, y_{0}, \alpha$ y $u$, las coordenadas de los cuatro vértices del recuadro se toman igual a:

1) $\left(x_{0}, y_{0}\right)$

2) $\left(x_{0}+u \cos \alpha, y_{0}+u \operatorname{sen} \alpha\right)$

3) $\left[x_{0}+u \sqrt{2} \cos \left(\alpha+45^{\circ}\right), y_{0}+u \sqrt{2} \operatorname{sen}\left(\alpha+45^{\circ}\right)\right]=\left(x_{0}+u \cos \alpha-u \operatorname{sen} \alpha\right.$, $\left.y_{0}+u \operatorname{sen} \alpha+u \cos \alpha\right)$

4) $\left[x_{0}+u \cos \left(\alpha+90^{\circ}\right), y_{0}+u \operatorname{sen}\left(\alpha+90^{\circ}\right)\right]=\left(x_{0}-u \operatorname{sen} \alpha, y_{0}+u \cos \alpha\right)$

Para un recuadro dado, el vector $\bar{V}$ puede elegirse de varias maneras diferentes, equivalentes entre sí; pero cada una de ellas determina unívocamente al recuadro en cuestión (3).

La posición de una etiqueta $E=\left\{r_{i} / 1 \leqslant i \leqslant n\right\}$ puede definirse mediante el conjunto de las de sus recuadros, $\left\{\bar{V}_{i} / 1 \leqslant i \leqslant n\right\}$. Pero, dado que todos ellos son del mismo tamaño y orientación y con posiciones relativas fijas, hace falta realmente un número inferior de parámetros: basta dar $\bar{V}_{1}$ (vector posición correspondiente al primer recuadro, o recuadro de referencia) y dos vectores enteros (de términos positivos, nulos o negativos): $J^{\prime}{ }_{k}, J^{\prime \prime}{ }_{k}(1 \leqslant k \leqslant$

(3) Fijando un sentido de recorrido determinado del contorno del recuadro, se tienen evidentemente cuatro elecciones posibles de $\overline{\mathrm{V}}$; y otras cuatro si el sentido de recorrido es opuesto (las fórmulas [2.2] presuponen un sentido de giro contrario al de las agujas del reloj). 
$\leqslant m)$, tales que indiquen el número de recuadros a ambos lados de una recta que pase por los vértices 1-4 del recuadro de referencia, en sucesivas «filas» $k=1,2, \ldots, m$. De modo más preciso, los valores de $x_{0 i}, y_{0 i}(1 \leqslant i \leqslant m)$ de los sucesivos recuadros $r_{i}$ de la etiqueta se obtienen mediante las fórmulas:

$$
\begin{aligned}
& x_{0 i}=x_{01}-(k-1) u \operatorname{sen} \alpha+j u \cos \alpha \\
& y_{0 i}=y_{01}+(k-1) u \cos \alpha+j u \operatorname{sen} \alpha,
\end{aligned}
$$

haciendo variar los índices enteros $k$ y $j$ entre los límites siguientes:

$$
\begin{gathered}
-J^{\prime}{ }_{k} \leqslant j \leqslant J^{\prime \prime}{ }_{k}-1 \\
1 \leqslant k \leqslant m
\end{gathered}
$$

Entonces, evidentemente:

$$
n=\sum_{k=1}^{m}\left(J_{k}^{\prime}+J_{k}^{\prime \prime}\right)
$$

Como el recuadro de referencia $\left(\bar{V}_{1}\right)$ se obtiene con $k=1$ y $j=0$, debe pertenecer a la fila $k=1$ (no puede situarse en una fila intermedia).

Así, por ejemplo, los índices ${J^{\prime}}_{k}$ y $J^{\prime \prime}{ }_{k}$ correspondientes a la etiqueta de la figura 1, son:

$$
\begin{array}{ll}
J^{\prime}=1 & J^{\prime \prime}{ }_{1}=3 \\
J^{\prime}{ }_{2}=1 & J^{\prime \prime}{ }_{2}=0 \\
J^{\prime}=-1 & J^{\prime \prime}{ }_{3}=4 \\
J^{\prime}{ }_{4}=2 & J^{\prime \prime}{ }_{4}=2
\end{array}
$$

\section{MEMORIA DE POSICIONES OCUPADAS}

Evidentemente, para poder precisar el estado de ocupación de los infinitos puntos de la zona conflictiva del plano (4), haría falta una cantidad infinita de información. Por ello es necesario discretizar dicha zona conflictiva; y considerar cada elemento de la misma como una unidad, a efectos de su estado de ocupación. Aquí supondremos que dichos elementos son cuadrados de lados paralelos a los ejes coordenados, y que el lado de dichos cuadrados es constante para todos ellos $\left(u^{\prime}\right)$; igual que en la delimitación de una etiqueta, un valor pequeño de $u^{\prime}$ mejora la adaptación al dominio a aproximar (ahora, la zona conflictiva completa), pero aumenta la cantidad de información necesaria para describir su estado de ocupación, así como los tiempos de cálculo.

Para mayor sencillez, supondremos que la zona conflictiva es rectangular (o que se elige como tal un rectángulo que la contenga), con dimensiones $m_{0} u^{\prime}, n_{0} u^{\prime}\left(m_{0}\right.$ y $n_{0}=$ números enteros positivos); entonces la partición en elementos puede hacerse tal como se indica en la figura 2. Siempre que los ejes coordenados y la numeración de los elementos se establezcan como en dicha figura, las coordenadas del centro de cada elemento valen:

$$
x_{i j}=\left(i-\frac{1}{2}\right) u^{\prime} \quad ; \quad y_{i j}=\left(j-\frac{1}{2}\right) u^{\prime}
$$

variando $i$ entre 1 y $m_{0} ;$ y $j$ entre 1 y $n_{0}$. En lo sucesivo denominaremos recuadro-base a cada elemento $\left(r_{i j}^{\prime}\right)$ de la zona conflictiva y malla-base al conjunto de los mismos.

(4) En el apartado precedente se ha considerado innecesario especificar el hecho obvio de que dicha zona es un dominio finito del espacio puntual euclídeo $\xi^{2}$; sin embargo, a pesar de sus dimensiones finitas, es bien sabido que un tal dominio contiene un número infinito (no numerable) de puntos. 




Fig. 2. Malla-base.

El estado de ocupación de un cierto recuadro-base $r^{\prime}$ es representable mediante una variable binaria $B$, con valor $B=1$ si el recuadro está "ocupado» y $B=0$ si está «libre» (la elección recíproca de valores de $B$ sería, desde luego, también válida). El conjunto de valores

$$
\left\{B_{i j} / 1 \leqslant i \leqslant m_{0}, 1 \leqslant j \leqslant n_{0}\right\}
$$

define el estado de ocupación de la malla-base. De momento no nos ocuparemos del criterio utilizado para llegar a definir todos esos valores $B_{i j}$, sino de la forma práctica de almacenar esa información en la memoria de un ordenador; esta memoria normalmente se organiza en forma de «palabras» $(\omega)$, o sea, en forma de conjuntos de $n_{b}$ «bits» $(b)$ :

$$
\omega=\left\{b_{m} / 0 \leqslant m \leqslant n_{b}-1\right\}
$$

Los dos estados o valores de cada bit (indicados también con la notación 1, 0) nos pueden servir para identificar los de cada $B_{i j}$; para ello será preciso utilizar en total los bits de $n_{\omega}$ palabras $\omega_{1}, \omega_{2}, \ldots$ :

$$
\omega_{l}=\left\{b_{l m} / 0 \leqslant m \leqslant n_{b}-1\right\} ; 1 \leqslant l \leqslant n_{\omega}
$$

Es decir, pondremos $b_{l m}=B_{i j}$, definiendo previamente una correspondencia unívoca entre indices $(i, j)$ y $(l, m)$ :

$$
(i, j) \rightarrow\left[\begin{array}{l}
l=1+\text { parte entera de } \frac{k-1}{n_{b}} \\
m=(k-1)-(l-1) n_{b}
\end{array}\right]
$$

siendo:

$$
k=(i-1) n_{0}+j\left(1 \leqslant k \leqslant m_{0} \cdot n_{0}\right)
$$


Naturalmente, otras correspondencias diferentes de la 3.4 son admisibles. Obviamente, el número de palabras $\left(n_{\dot{\omega}}\right)$ utilizadas debe verificar:

$$
n_{\dot{\omega}} \geqslant \frac{m_{0} \cdot n_{0}}{n_{b}}
$$

O, dicho de otro modo, con $n_{\omega}$ palabras el área máxima de la malla-base es $n_{\omega} n_{b} u^{\prime 2}$. Así, con $u^{\prime}=3 \times 10^{-3} \mathrm{~m}$ (recuadro-base de $3 \mathrm{~mm}$ de lado), se requieren algo menos de 7.000 palabras de 16 bits $\left(n_{\omega} \leqslant 7.000, n_{b}=16\right)$ por cada $\mathrm{m}^{2}$ de zona controlada de plano. Esto indica que, con ordenadores de capacidad media, la totalidad de las $n_{\omega}$ palabras puede tenerse normalmente en memoria, durante la ejecución del plano, de un modo permanente o casi permanente; si el plano es de gran extesión, puede ser necesario hacer uso de una memoria periférica (disco o cinta magnética), con transferencias a la memoria central de la parte requerida en cada instante.

Nótese que el planteamiento anterior requiere trabajar a nivel de bit; pues utilizar una palabra completa como almacén de cada $B_{i j}$, exigiría multiplicar por $n_{b}$ la capacidad de memoria necesaria. El lenguaje FORTRAN no permite el acceso directo a los $n_{b}$ bits de cada palabra; ello obliga, para dicho acceso, a realizar (cada vez) un conjunto de operaciones aritméticas no excesivamente extenso, pero que llega a repetirse un número de veces muy elevado en cada utilización del programa. Por eso es de gran interés disponer de una o varias subrutinas (5), escritas en un lenguaje más primario (6), que permitan «leer»o "alterar» (7) de un modo directo el contenido del bit $m$-ésimo $\left(0 \leqslant m \leqslant n_{b}-1\right)$ de una palabra dada.

\section{4. $^{\circ}$ CRITERIO DE OCUPACION}

Veamos ahora cuándo consideraremos «ocupado» un cierto recuadro-base $r^{\prime}$ por un recuadro $r$ dado (definido por un vector del tipo 2.1). Es decir, definamos una función $F\left(r, r^{\prime}\right)$ que valga 1 si efectivamente $r^{\prime}$ es ocupado por $r$; y 0 en caso contrario. Este criterio de ocupación (básico a todos los efectos) puede elegirse de múltiples formas, pero atendiendo a lo siguiente:

a) Debe ser de la máxima rapidez, ya que en cada utilización del programa se apela un gran número de veces a dicho criterio de ocupación.

b) Debido a la discretización del plano en recuadros-base (cada uno, considerado con un estado de ocupación uniforme en su interior), no es posible garantizar la ausencia absoluta de interpenetración entre recuadros móviles sin exigir a su vez, en ocasiones, ciertas separaciones libres entre los mismos. Por ello es conveniente que el criterio de ocupación incluya un parámetro ajustable, que permita llegar a una solución de compromiso entre las penetraciones y separaciones aleatorias entre recuadros.

Un posible criterio de ocupación consiste en poner $F\left(r, r^{\prime}\right)=1$ si $r \cap r^{\prime} \neq \phi$ (intersección de $r$ y $r^{\prime}$, no vacía) y $F\left(r, r^{\prime}\right)=0$ si $r \cap r^{\prime}=\phi$ (ausencia de puntos comunes); pero es fácil ver que esto conduce a separaciones medias excesivas entre etiquetas (8). Una solución mejor es poner $F\left(r, r^{\prime}\right)=1$ si la relación (área de $\left.r \cap r^{\prime}\right) /\left(\right.$ área de $r^{\prime}$ ) es mayor que un cierto tanto por uno ( $F=0$ si es menor); pero el cálculo del área de $r \cap r^{\prime}$ es relativamente laborioso.

(5) Compatibles con los respectivos "CALL" del programa principal (éste, normalmente escrito en un cierto dialecto del FORTRAN).

(6) Ensamblador.

(7) O sea, transferir su contenido (1 ó 0) a una variable FORTRAN, o viceversa.

(8) Aunque el posicionamiento de cada etiqueta no se comenta hasta la sección siguiente, es obvio que a lo que vamos es a no admitir una determinada posición de la etiqueta si alguno de sus recuadros ocupa algún recuadro-base previamente ocupado por alguna otra etiqueta anterior. 


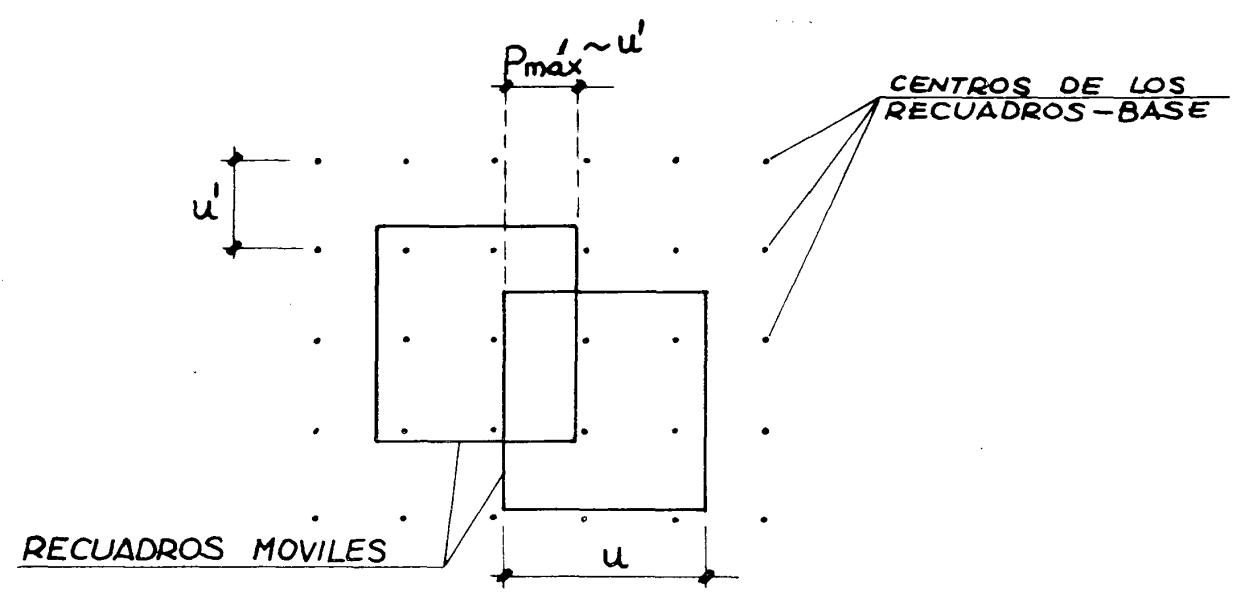

Fig. 3. Recuadros estrictamente compatibles $\operatorname{con} \mathbf{q}=\mathbf{1}$.

Otra posible solución consiste en poner $F\left(r, r^{\prime}\right)=1$ si y sólo si el centro de $r^{\prime}$ es interior a $r$; pero esto admite (ver fig. 3) penetraciones del orden de $u^{\prime}$ entre recuadros móviles, lo cual supone un aprovechamiento deficiente de la malla-base, requiriendo reducir $u^{\prime}$ de forma excesiva.

La solución anterior tiene, sin embargo, el atractivo de que es muy rápido averiguar si un punto es interior a un cierto cuadrado. Por ello es la solución que adoptaremos, aunque modificada: sustituiremos (a efecto únicamente de este control) el recuadro $r$ por un recuadro ampliado, del mismo centro y orientación, pero de lado $q u>u$. Entonces:

$$
\begin{aligned}
& F\left(r, r^{\prime}\right)=1 \rightleftarrows\left[0<\Delta x^{\prime}<q u \quad \text { y } \quad 0<\Delta y^{\prime}<q u\right] \\
& F\left(r r^{\prime}\right)=0 \rightleftarrows\left[\Delta x^{\prime} \leqslant 0 \text { ó } \Delta x^{\prime} \geqslant q u \text { ó } \Delta y^{\prime} \leqslant 0 \quad \text { ó } \quad \Delta y^{\prime} \geqslant q u\right]
\end{aligned}
$$

calculándose $\Delta x^{\prime}$ e $\Delta y^{\prime}$ en función de $x, y$ (coordenadas del centro de $r^{\prime}$ ) y de $x_{0}, y_{0}, \alpha, u$ (parámetros definidores de $r$ ) según las fórmulas siguientes:

$$
\begin{aligned}
& x_{0}{ }^{*}=x_{0}-(\cos \alpha-\operatorname{sen} \alpha)(q-1) u / 2 \\
& y_{0}{ }^{*}=y_{0}-(\cos \alpha+\operatorname{sen} \alpha)(q-1) u / 2 \\
& \Delta x=x-x_{0}{ }^{*} \quad ; \quad \Delta y=y-y_{0}{ }^{*} \\
& \Delta x^{\prime}=\cos \alpha \Delta x+\operatorname{sen} \alpha \Delta y \\
& \Delta y^{\prime}=-\operatorname{sen} \alpha \Delta x+\cos \alpha \Delta y
\end{aligned}
$$

La elección del parámetro $q$ debe hacerse, de acuerdo con lo dicho en el párrafo $b$ ), de modo que la probabilidad de penetraciones inaceptables entre etiquetas sea pequeña o nula, pero sin exigir separaciones excesivas entre recuadros estrictamente compatibles. Un estudio riguroso del problema se expone en el apartado 6 , y conduce a valores de $q$ variables con la relación $\lambda=u / u^{\prime}$; así, para $u^{\prime}=3 \mathrm{~mm}$ y $u=4 \mathrm{~mm}$, una elección razonable resulta ser $q=$ $=1,5$, cuando se admiten pequeñas interpenetraciones de las etiquetas; con $q=1,75$, éstas se eliminan por completo.

\section{POSICIONAMIENTO DE UNA ETIQUETA}

En primer lugar, establezcamos el criterio de aceptación o rechazo de una determinada posición de una cierta etiqueta; luego nos ocuparemos de la estrategia a seguir en caso de que sea inaceptable la posición establecida a priori como óptima. Suponemos definido un estado de ocupación $\left\{B_{i j}\right\}$ dado, debido a las etiquetas y demás elementos dibujados con anterioridad a la etiqueta en cuestión. 
Aceptaremos una posición dada de la etiqueta $E=\left\{r_{k} / 1 \leqslant k \leqslant n\right\}$ si se verifica $B_{i j}=0$ para todo $r_{i j}^{\prime}$ tal que $F\left(r_{k}, r_{i j}^{\prime}\right)=1$; y la rechazaremos si existe al menos una pareja $r_{i j}^{\prime}$ y $r_{k}$ tales que $B_{i j}=1$ y $F\left(r_{k}, r_{i j}^{\prime}\right)=1$. Es decir, aceptaremos la etiqueta si y sólo si todos sus recuadros ocupan únicamente recuadros-base libres.

Si la posición de la etiqueta es aceptada, se procede a su dibujo y a la ocupación de todos los recuadros-base ocupados por cada uno de los $r_{k}$ pertenecientes a $E$ (asignación del valor 1 a los correspondientes $B_{i j}$ ). Nótese que esta «ocupación» se efectúa después de haber verificado la aceptación de las posiciones de todos los $r_{k}$ de $E$, y no a medida que se van chequeando los sucesivos $r_{k}$; en efecto, eso debe ser así, ya que hasta no haber verificado la totalidad de los $r_{k}$ de $E$ no se sabe si la posición de $E$ es válida; y porque admitimos, sin más, que dos recuadros cualesquiera de $E$ son siempre compatibles entre sí (9).

En la práctica, para investigar la posible aceptación de una etiqueta, no es necesario chequear cada uno de sus recuadros con todos los $m_{0} n_{0}$ recuadros-base $r^{\prime}{ }_{i j}$. En efecto, fijado un $r$ dado, es inmediato establecer un conjunto de $r_{i j}^{\prime}$ relativamente limitado que contenga a todos los posibles $r_{i j}^{\prime}$ para los cuales $F\left(r, r_{i j}^{\prime}\right) \neq 0$ [«soporte» de $F_{r}\left(r^{\prime}\right) \equiv F\left(r, r^{\prime}\right)$ ]. En efecto, si $\bar{V}^{t}=\left[x_{0}, y_{0}, \alpha, u\right]$ es el vector-posición de $r$, basta chequear los $r^{\prime}{ }_{i j}$ tales que $i_{1} \leqslant i \leqslant i_{2}$, $j_{1} \leqslant j \leqslant j_{2}$, siendo:

$$
\begin{aligned}
& i_{1}=\text { parte entera } \mathrm{de}\left(\frac{1}{2}+\frac{x_{\text {min }}^{*}}{u^{\prime}}\right)\left(i_{1} \nless 1\right) \\
& j_{1}=\text { parte entera } \operatorname{de}\left(\frac{1}{2}+\frac{y_{\text {min }}^{*}}{u^{\prime}}\right)\left(j_{1} \nless 1\right) \\
& i_{2}=\text { menor número entero mayor o igual que }\left(\frac{1}{2}+\frac{x_{\text {max }}^{*}}{u^{\prime}}\right)\left(i_{2} \ngtr m_{0}\right) \\
& j_{2}=\text { menor número entero mayor o igual que }\left(\frac{1}{2}+\frac{y_{\text {max }}^{*}}{u^{\prime}}\right)\left(j_{2} \ngtr m_{0}\right)
\end{aligned}
$$

siendo $x_{\text {min }}^{*}, x^{*}{ }_{\text {max }}, y^{*}{ }_{\text {min }}, y^{*}{ }_{\max }$ los valores extremos de las coordenadas del recuadro «ampliado»; o sea, de las coordenadas calculables mediante las fórmulas [2.2], sustituyendo $u$ por $q u$ y $x_{0}, y_{0}$ por $x_{0}^{*}, y_{0}^{*}$ (ver apartado $\left.4 .^{\circ}\right)$.

Hablemos ahora de la posible estrategia a seguir cuando la posición más deseable, establecida a priori, resulta inaceptable. Para gran cantidad de problemas basta tantear traslaciones del conjunto de la etiqueta en las direcciones paralela y perpendicular a la propia orientación de la misma; otras veces conviene más tantear posiciones centradas sobre una circunferencia en torno a un punto dado (con radios crecientes si es preciso), normalmente manteniendo siempre invariable el valor de $\alpha$ (en ocasiones pueden también imprimirse giros de conjunto). Veamos con más detalle el primer caso, en que simplemente se aplican traslaciones de orientación igual a $\alpha, \alpha+180^{\circ}$ ó $\alpha \pm 90^{\circ}$; entonces, de $\bar{V}_{1}^{t}=\left[x_{01}, Y_{01}, \alpha, u\right], \bar{J}^{\prime}$ y $\bar{J}^{\prime \prime}$ (parámetros posicionadores de la etiqueta, definidos en el apartado $2 .^{\circ}$ ), sólo varían $x_{01}$ e $y_{01}$, los cuales experimentan las siguientes modificaciones respecto a sus valores iniciales:

$$
\begin{aligned}
& \Delta x_{01}=a \cos \alpha-b \operatorname{sen} \alpha \\
& \Delta y_{01}=a \operatorname{sen} \alpha+b \cos \alpha
\end{aligned}
$$

Normalmente $a$ y $b$ se modulan respecto de $u: a=0, \pm u, \pm 2 u, \pm 3 u, \ldots ;$ y análogamente para $b$. También puede interesar tantear posiciones progresivamente más alejadas de la inicial; por ejemplo, $a= \pm 0,75 \cdot i^{3 / 2}$, con $i=0,1,2,3, \ldots$ En estas secuencias de valores de $a$ y $b$ se limita el número de términos (limitación de la separación respecto a la posición óptima); a veces hay que prescribir también un signo determinado: por ejemplo, $b \leqslant 0$ si la etiqueta no puede moverse por «encima» de la posición inicial. Se combinan todos los valores prescritos de $a$ con todos los de $b$, hasta encontrar una posición válida.

(9) En cambio, dado lo imperfecto de los criterios de ocupación propuestos en el apartado 4. ${ }^{\circ}$ (imperfección debida al carácter discreto de la malla-base), algunos de dichos criterios podrían dar en ocasiones como incompatibles a dos recuadros (diferentes) de la misma etiqueta. 
Si se agotan todos los tanteos previstos, o las separaciones respecto de la posición inicial son excesivas, conviene detener el proceso y proceder al dibujo de la etiqueta al margen del plano, refiriéndola mediante unas siglas adecuadas (tarea que puede realizar el propio programa).

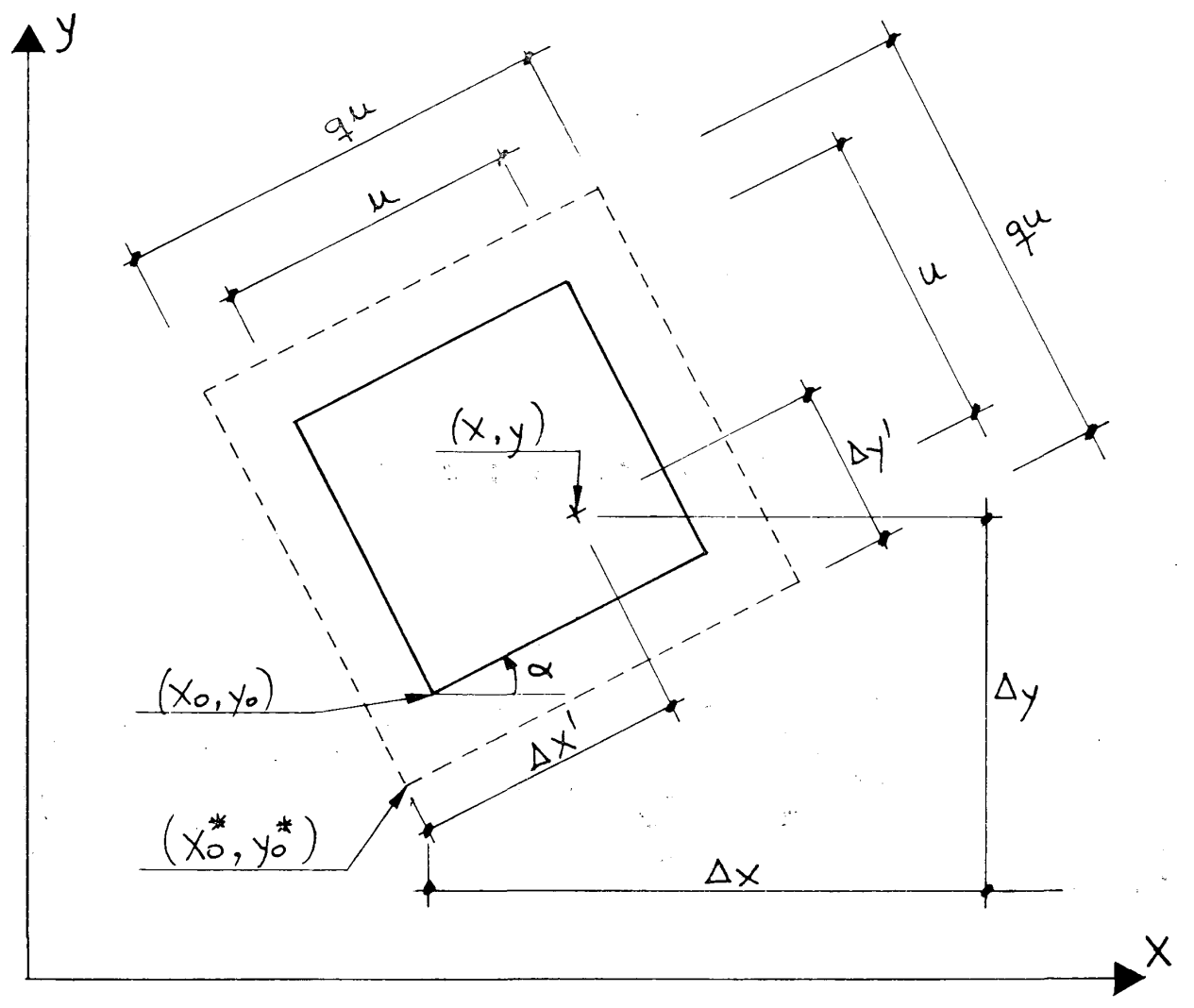

Fig. 4. Recuadro ampliado.

\section{TEORIA DEL CONTROL OCUPACIONAL DISCRETO EN UNA DIMENSION}

El objeto de la presente sección es el de estudiar el nivel de eficiencia del criterio de ocupación propuesto en el apartado 4. Para mayor sencillez se estudia sólo el caso unidimensional; en el problema bidimensional valen los mismos resultados para las direcciones $x$ e $y$ independientemente, siempre que la orientación de los recuadros sea paralela a los ejes coordenados; con orientaciones arbitrarias (como es nuestro caso), dichos resultados son tan sólo aproximados.

Consideremos, pues, un intervalo $0<x<m_{0} u^{\prime}$, en el que se quiere efectuar un control ocupacional haciendo uso de una cantidad de información igual a $m_{0}$ bits $\left(B_{i}, 1 \leqslant i \leqslant m_{0}\right)$. Definimos a ese efecto una malla de intervalos-base:

siendo:

$$
r_{i}^{\prime} \equiv\left(x_{i}^{\prime}-\frac{u^{\prime}}{2}, x_{i}^{\prime}+\frac{u^{\prime}}{2}\right)
$$

$$
x_{i}^{\prime}=\left(i-\frac{1}{2}\right) u^{\prime} \quad ; \quad 1 \leqslant i \leqslant m_{0} ;
$$

pondremos $B_{i}=1$ si $r_{i}^{\prime}$ está «ocupado» y $B_{i}=0$ si está «libre».

Con ese control pretendemos que, para sucesivos «intervalos móviles»

$$
r_{x, u} \equiv\left(x-\frac{u}{2}, x+\frac{u}{2}\right)
$$


pueda cada uno de ellos rechazarse o aceptarse según que interactúe o no desfavorablemente con los precedentes (que han modificado el estado de ocupación $\left\{B_{i}\right\}$, partiendo de 0 ). Un intervalo dado se acepta sólo si todos los intervalos-base ocupados por él están libres. El criterio de ocupación lo establecemos en función de un parámetro fijo $q \geqslant 1$ :

$$
r_{i}^{\prime} \text { es ocupado por } r_{x, u} \rightleftarrows x_{i}^{\prime} \text { pertenece a } r_{x, q u} \rightleftarrows\left|x_{i}^{\prime}-x\right|<\frac{q u}{2}
$$

Si se acepta un determinado intervalo $r_{x, u}$, se hace $B_{i}=1$ para todos los $r_{i}^{\prime}$ ocupados por el mismo; y a continuación se procede a estudiar el siguiente intervalo móvil.

Debido al carácter finito de la malla-base, puede suceder que se consideren compatibles dos intervalos $r_{x_{1}, u}$ y $r_{x_{2}, u}$ cuya intersección $r_{x_{1}, u} \cap r_{x_{2}, u}$ sea no vacía; es decir, dos intervalos compatibles pueden tener una «penetración» $p \neq 0\left(p=\right.$ longitud del intervalo $\left.r_{x_{1}, u} \cap r_{x_{2}, u}\right)$. Recíprocamente, pueden darse como incompatibles dos intervalos entre los que exista una separación libre $s>0$. Es evidente que:

$$
\begin{aligned}
& p=u-\left|x_{2}-x_{1}\right| \text { si }\left|x_{2}-x_{1}\right|<u \quad ; \quad p=0 \text { en caso contrario } \\
& s=\left|x_{2}-x_{1}\right|-u \text { si }\left|x_{2}-x_{1}\right|>u ; s=0 \text { en caso contrario }
\end{aligned}
$$

Por conveniencia en los cálculos, consideraremos $s$ como una penetración negativa; entonces podemos escribir, en cualquier caso:

$$
p=u-\left|x_{2}-x_{1}\right|
$$

Si consideramos la aparición de los dos intervalos $r_{x_{1}, u}$ y $r_{x_{2}, u}(u=$ constante) como sucesos aleatorios con valores equiprobables de $x_{1}$ y $x_{2}$ en $\left(u / 2, m_{0} u^{\prime}-u / 2\right)$, con la única restricción de que ambos intervalos sean compatibles (según nuestro criterio ocupacional discreto), entonces $p$ y $s$ se convierten a su vez en variables aleatorias. El estudio de las mismas nos permitirá sacar conclusiones acerca de la mayor o menor eficiencia del criterio de ocupación adoptado.

Sea $I_{x, u}$ el conjunto de índices $\left\{i_{1}, i_{1}+1, \ldots, i_{2}\right\}$ de los intervalos-base ocupados por un $r_{x, u}$ dado. Entonces:

$$
\begin{aligned}
& x-\frac{q u}{2}=\left(i_{1}-\frac{1}{2}\right) u^{\prime}-\varepsilon_{1} u^{\prime} \quad ; \quad 0<\varepsilon_{1} \leqslant 1 \\
& x+\frac{q u}{2}=\left(i_{2}-\frac{1}{2}\right) u^{\prime}+\varepsilon_{2} u^{\prime} \quad ; \quad 0<\varepsilon_{2} \leqslant 1
\end{aligned}
$$

Consideramos $r_{x_{1}, u}$ y $r_{x_{2}, u}$ compatibles cuando $I_{x_{1}, u} \cap I_{x_{2}, u}=\phi$; o sea, cuando (10) $i_{22} \leqslant i_{11}-1$ ó $i_{12} \geqslant i_{21}+1$. Pero:

$$
\begin{aligned}
& i_{22} \leqslant i_{11}-1 \rightleftarrows \frac{1}{u^{\prime}}\left(x_{2}+\frac{q u}{2}\right)-\varepsilon_{22} \leqslant \frac{1}{u^{\prime}}\left(x_{1}-\frac{q u}{2}\right)+\varepsilon_{11}-1 \rightleftarrows x_{1}-x_{2} \geqslant q u- \\
& -\left(\varepsilon_{11}+\varepsilon_{22}-1\right) u^{\prime} \\
& i_{12} \geqslant i_{21}+1 \leftrightarrows \frac{1}{u^{\prime}}\left(x_{2}-\frac{q u}{2}\right)+\varepsilon_{12} \geqslant \frac{1}{u^{\prime}}\left(x_{1}+\frac{q u}{2}\right)-\varepsilon_{21}+1 \rightleftarrows x_{2}-x_{1} \geqslant q u- \\
& \left(\varepsilon_{12}+\varepsilon_{21}-1\right) u^{\prime}
\end{aligned}
$$

Definamos:

$$
\begin{gathered}
\lambda=u / u^{\prime} \\
t=(q-1) / 2 \\
\tau_{1}=\frac{1}{2}\left(\varepsilon_{11}+\varepsilon_{22}-1\right) \quad ; \quad \tau_{2}=\frac{1}{2}\left(\varepsilon_{12}+\varepsilon_{21}-1\right)
\end{gathered}
$$

(10) Poniendo $i_{11}, i_{21}, \varepsilon_{11}, \varepsilon_{21}$ para $I_{x_{1}},{ }_{u}$; e $i_{12}, i_{22}, \varepsilon_{12}, \varepsilon_{22}$ para $I_{x_{2}}, u$. 
[6.5] $\left(i_{22}<i_{11}\right)$ corresponde normalmente a situaciones en las que $x_{2}<x_{1}$ (11), en cuyo caso $x_{1}-x_{2}=\left|x_{2}-x_{1}\right|$. Análogamente, en [6.6], $x_{2}-x_{1}=\left|x_{2}-x_{1}\right|$; con esto y con las definiciones [6.7], [6.8] y [6.9], resulta como criterio de compatibilidad de los dos intervalos, el siguiente:

$$
p \leqslant 2\left(\frac{\tau}{\lambda}-t\right) u
$$

siendo $\tau$ cualquiera de las dos variables aleatorias $\tau_{1}$ ó $\tau_{2}$, que poseen la misma distribución de probabilidad. Es fácil ver que esta distribución es de la forma indicada en la figura 5 . En efecto, las $\varepsilon_{11}, \varepsilon_{21}, \varepsilon_{12}, \varepsilon_{22}$ tienen distribuciones prácticamente equiprobables en el intervalo $0<\varepsilon \leqslant 1$, es decir, con densidad de probabilidad uniforme igual a 1 en dicho intervalo (0 en el resto); luego $\varepsilon_{11}+\varepsilon_{22}$ (análogamente para $\varepsilon_{12}+\varepsilon_{21}$ ), suponiendo independientes entre sí $\varepsilon_{11}$ y $\varepsilon_{22}(12)$, aparece con función de distribución $G \varepsilon\left(\varepsilon_{11}+\varepsilon_{22}\right)$, siendo:

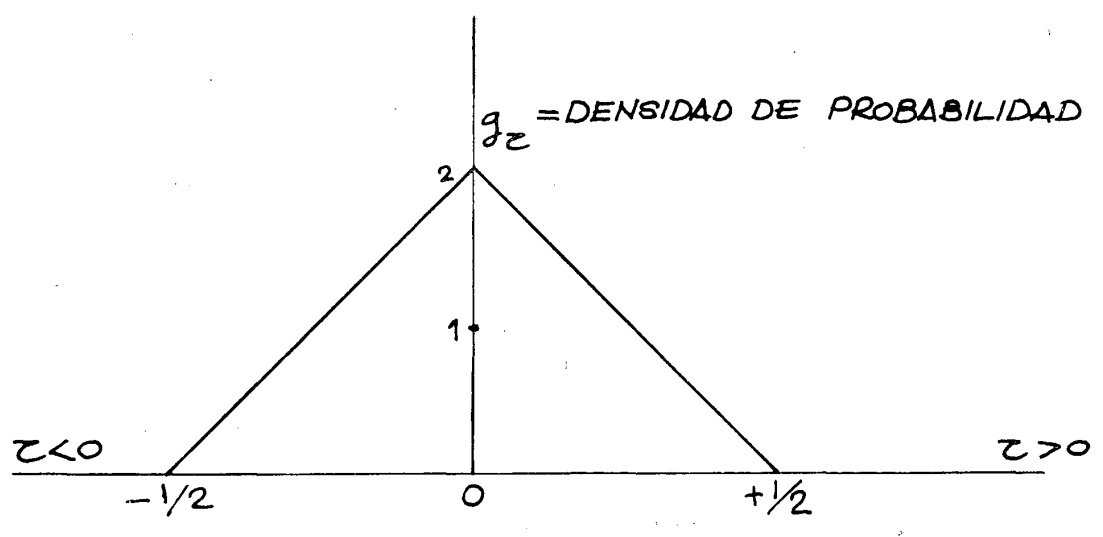

Fig. 5

$$
\begin{array}{ll}
G_{\varepsilon}(\varepsilon)=0 & \text { si } \quad \varepsilon \leqslant 0 \\
G_{\varepsilon}(\varepsilon)=\frac{\varepsilon^{2}}{2} & \text { si } 0<\varepsilon \leqslant 1 \\
G_{\varepsilon}(\varepsilon)=\frac{\varepsilon^{2}}{2}-\varepsilon^{2 / 2}-1 & \text { si } 1<\varepsilon \leqslant 2 \\
G_{\varepsilon}(\varepsilon)=1 & \text { si } \quad \varepsilon>2
\end{array}
$$

La obtención de dicha función de distribución $G_{\varepsilon}$ no ofrece dificultad (13). De la misma se deduce la siguiente función densidad de probabilidad $g_{\varepsilon}=d G_{\varepsilon}(\varepsilon) / d \varepsilon$ :

$$
\begin{aligned}
& G_{\varepsilon}(\varepsilon)=0 \quad \text { si } \quad \varepsilon \leqslant 0 ; g_{\varepsilon}(\varepsilon)=\varepsilon \quad \text { si } 0<\varepsilon \leqslant 1 \\
& g_{\varepsilon}(\varepsilon)=2-\varepsilon \text { si } 1<\varepsilon \leqslant 2 ; g_{\varepsilon}(\varepsilon)=0 \text { si } \varepsilon>2
\end{aligned}
$$

(11) Si $\lambda<1 / q$, existe una probabilidad no nula de que eso no sea cierto, cosa que ignoramos con el fin de no complicar excesivamente el análisis; 0 , de otro modo, nos limitamos al caso en que $\lambda q \geqslant 1$. Para ello es suficiente que $u \geqslant u^{\prime}$ y $q \geqslant 1$.

(12) El estudio de la variable aleatoria $\varepsilon_{11}+\varepsilon_{22}$ es previo a la restricción de compatibilidad mutua entre ambos intervalos. Es decir, estudiamos $\varepsilon_{11}+\varepsilon_{22}$ en la hipótesis de que $r_{x 1},{ }_{1}, r_{x 2}$, aparecen de forma independiente, en cuyo caso también $\varepsilon_{11}$ y $\varepsilon_{22}$ son independientes entre sí. De todas formas, como normalmente $m_{0} u^{\prime} \gg u$, la exclusión de los intervalos incompatibles (pero considerando toda la gama de intervalos compatibles, no sólo los estrictamente compatibles) apenas afecta a $G_{\varepsilon}\left(\varepsilon_{11}+\varepsilon_{22}\right)$.

(13) Dado que el punto $\left(\varepsilon_{11}, \varepsilon_{22}\right)$ aparece de forma equiprobable dentro del rectángulo $(0,1) \times(0,1)$, GE $(\varepsilon)$ es proporcional al área de la zona de dicho rectángulo que verifica $\varepsilon_{11}+\varepsilon_{22} \leqslant \varepsilon$. De ahí resulta [6.11] de forma inmediata. 


\section{Y para}

$$
\begin{aligned}
& \tau=(\varepsilon-1) / 2 \quad, \quad g_{\tau}(\tau)=g_{\varepsilon} \frac{d \varepsilon}{d \tau}=2 g_{\varepsilon}(1+2 \tau) \quad ; \quad \text { o sea: } \\
& g_{\tau}(\tau)=0 \quad \text { si } \quad \tau \leqslant-1 / 2 \\
& g_{\tau}(\tau)=2+4 \tau \quad \text { si }-1 / 2<\tau \leqslant 0 \\
& g_{\tau}(\tau)=2-4 \tau \text { si } 0<\tau \leqslant 1 / 2 \\
& g_{\tau}(\tau)=0 \quad \text { si } \quad \tau>1 / 2
\end{aligned}
$$

que es la expresión analítica correspondiente al gráfico de la figura 5.

El caso de mayor interés es el de intervalos estrictamente compatibles. Para los mismos, el signo $\leqslant$ de [6.10] se transforma en signo de igualdad:

$$
p=2\left(\frac{\tau}{\lambda}-t\right) u
$$

Puesto que $\tau$ es una variable aleatoria, deducimos que dos intervalos estrictamente compatibles según nuestros criterios presentan en realidad interpenetraciones aleatorias, o separaciones aleatorias (recordar que $p>0$ significa penetración mutua; y $p<0$, separación de valor $s=-p)$. Para conseguir que $p$ fuese idénticamente nula en el caso de compatibilidad estricta de intervalos móviles finitos («u» finita), se requeriría que $t=0(q=1)$ y que $\lambda \rightarrow \infty$; por lo tanto, que $u^{\prime} \rightarrow 0$. Pero ello no está a nuestro alcance, ya que exigiría memorias de dimensión infinita (14).

Según [6.14], se deduce que

$$
g_{p}=\frac{\lambda}{2 u} g_{\tau}\left[\left(\frac{p}{2 u}+t\right) \lambda\right]
$$

es decir, la función densidad de probabilidad representada en la figura 6. De la misma se infiere que:

Valor medio de $p: p_{\text {med }}=-2 t u=(1-q) u$

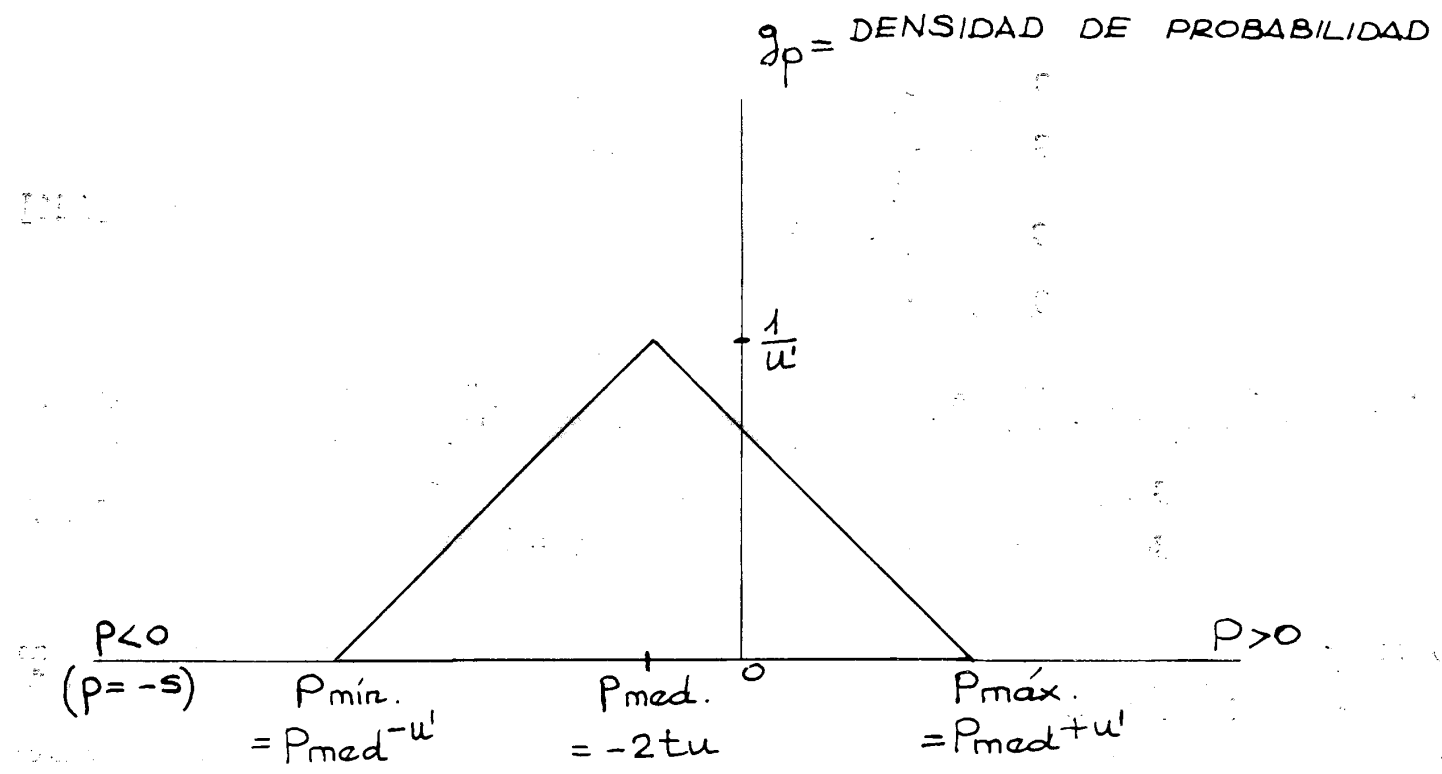

Fig. 6. Distribución de probabilidad de la penetración entre intervalos estrictamente compatibles.

(14) Sin embargo, el control "perfecto" indicado podría conseguirse con un infinito numerable de bits, a pesar de referirse a un infinito no numerable de puntos. 


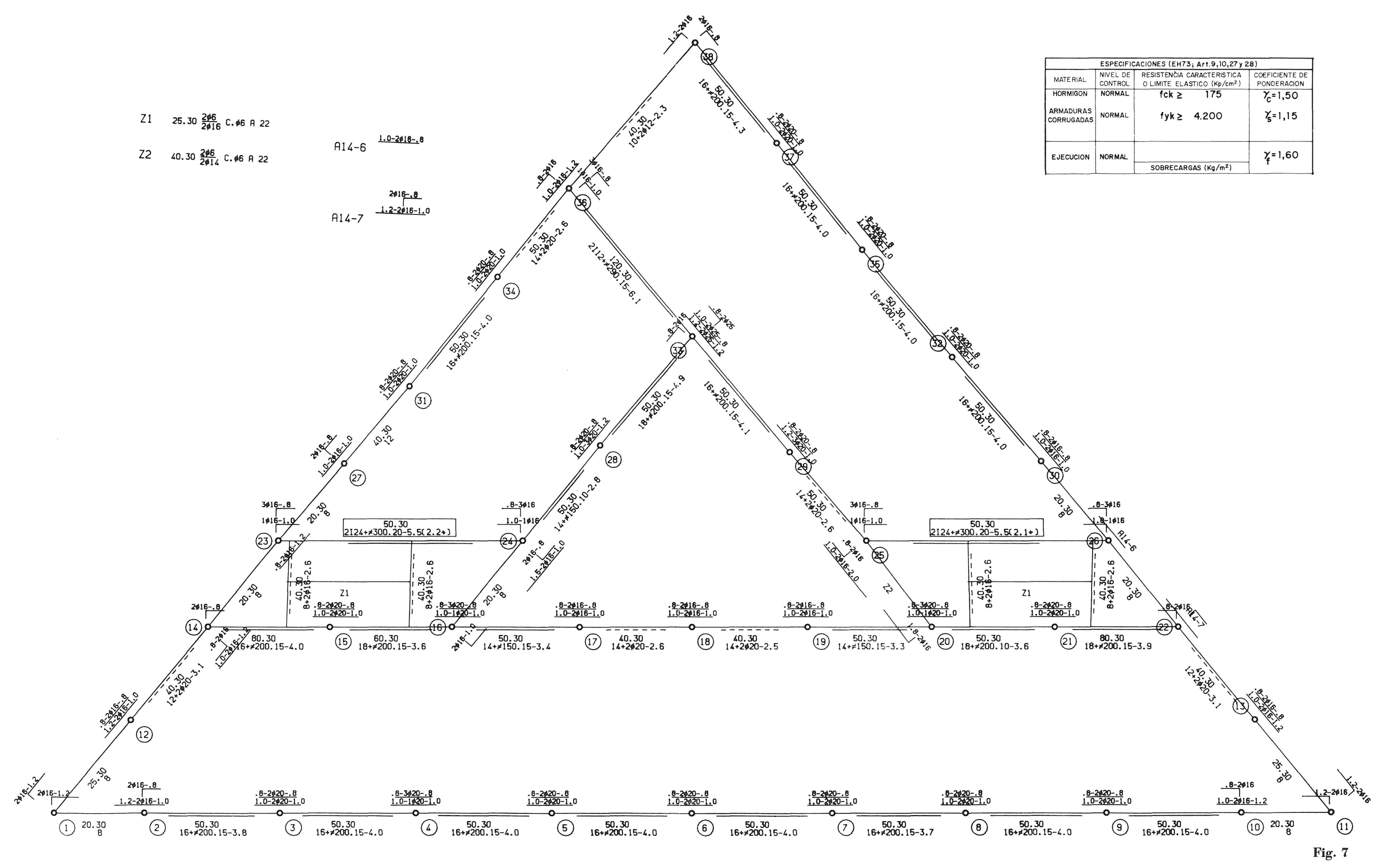


Valor más probable de $p: p_{\text {med }}$

Desviación típica: $\sigma_{p}=\mathcal{u}^{\prime} / \sqrt{6} \simeq \mathcal{u}^{\prime} / 2,45$

Valor máximo de $p: p_{\max }=\left(\frac{1}{2}-2 t\right) u=p_{\operatorname{med}}+u^{\prime}$

Valor mínimo de $p\left(=-s_{\max }\right): p_{\min }=-\left(\frac{1}{\lambda}+2 t\right) u=p_{\text {med }}-u^{\prime}$

$$
p_{\max }+s_{\max }=2 u^{\prime}
$$

Nótese que la elección del parámetro $q$ (o de $t$, ambos ligados a través de [6.8]) permite fijar un valor dado de $p_{\text {med }}$; pero que las diferencias $p_{\max }-p_{\text {med }}, p_{\text {med }}-p_{\min }$ dependen sólo de la dimensión de los recuadros-base $\left(u^{\prime}\right)$. Así, eligiendo

$$
q=1+\frac{1}{\lambda}\left(t=\frac{1}{2 \lambda}\right)
$$

conseguiremos que $p_{\max }=0$ (ausencia de penetraciones), pero a costa de que $s_{\max }=2 u^{\prime}$, según [6.16]. Si no se tiene una exigencia total de ausencia de superposiciones, puede ser preferible admitir valores no nulos de $p_{\max }$, lo cual permite disminuir algo $s_{\max }$. Por ejem plo, fijando de antemano la relación $\psi=p_{\max } / s_{\max }$, se deduce:

$$
q=1+\frac{(1-\psi)}{(1+\psi) \lambda}
$$

Así, con $u^{\prime}=3 \mathrm{~mm}, u=4 \mathrm{~mm}(\lambda=4 / 3)$ y $\psi=0,2$, se obtiene:

$$
\begin{aligned}
& q=1,5 \\
& p_{\text {med }}=-2 \mathrm{~mm}, p_{\max }=1 \mathrm{~mm}, s_{\max }=5 \mathrm{~mm},\left(p_{\text {med }}+\sigma_{p}\right)=-0,78 \mathrm{~mm}
\end{aligned}
$$

Es preciso resaltar que la penetración $p_{\max }$ es la máxima posible en la situación más crítica (intervalos estrictamente compatibles), presentándose raramente; así, en el caso [6.18] $\left(p_{\text {med }}+\sigma_{p}<0\right)$ la garantía de no penetración es muy elevada.

\section{7..$^{\circ}$ EJEMPLO PRACTICO}

Se adjunta el plano de estructura de una planta de edificio totalmente dimensionado y delineado por medio de los programas de ordenador aludidos en el apartado $1 .^{\circ}$ El tiempo total de procesamiento, incluyendo realización de memoria completa (de datos, esfuerzos y piezas dimensionadas), medición y planos es del orden de 20 minutos por planta-tipo, a par. tir exclusivamente de datos geométricos y de acciones exteriores; ello da una idea de la posible rentabilidad de estos procedimientos automáticos para la realización de proyectos de estructuras de edificación (16).

El hecho de que el plano sea relativamente simple (elegido así para mayor facilidad de su reproducción) no debe hacer suponer que el sistema deje de ser aplicable para plantas de mayor extensión o complejidad (contando con el «hardware» adecuado). En particular, en lo relativo al tema específico del artículo (resolución de zonas de delineación conflictiva), el ejemplo adjunto es de una complejidad media; pero también se resuelven satisfactoriamente planos más complicados.

Se observará el elevado grado de codificación alfanumérica del plano, factor altamente deseable para la realización de este tipo de planos (otros planos de estructura, como los de soportes, pantallas, etc., requieren mayor grado de expresión gráfica). No se entra aquí a comentar el significado de los diferentes símbolos del plano, ya que lo que se trata de resaltar es su correcta disposición y la ausencia de superposiciones entre etiquetas. Los valores de $u, u^{\prime}$ y $q$ utilizados han sido: $u=3,5 \mathrm{~mm}, u^{\prime}=3 \mathrm{~mm}$ y $q=1,5$.

(15) Se recuerda que, si $q \lambda<1$, estos resultados son sólo aproximados.

(16) Sin embargo, no hay que olvidar los tiempos de codificación y perforación de datos, que pueden ser imporSintes. 


\section{8. $\quad$ NOTACION}

En [8.1] se indica alguna terminología matemática elemental, utilizada para no restar agilidad a la exposición. En [8.2] se reúnen los símbolos más frecuentes en esta última.


$\bar{A}=\left[\begin{array}{c}A_{1} \\ A_{2} \\ \vdots \\ A_{n}\end{array}\right]:$ vector-columna de $n$ términos

$\bar{A}^{t}=\left[A_{1}, A_{2}, \ldots, A_{n}\right]$ : vector-fila (vector transpuesto $\operatorname{del} \bar{A}$ )

$x, y=$ coordenadas cartesianas del plano

$n=$ inclinación de una cierta recta respecto del eje $O X^{+}$(normalmente, $\alpha$ se supone en grados sexagesimales, para mayor claridad)

$i, i, k, l, m, n=$ indices enteros

$r^{\prime}=$ recuadro-base

$u^{\prime}=$ longitud del lado de cada recuadro-base

$m_{0} n_{0}=$ número de recuadros-base

$r=$ recuadro-móvil

$\bar{V}=$ «vector-posición» del mismo (conjunto de cuatro parámetros definiendo su posición, orientación y dimensión)

$u=$ longitud del lado de un recuadro móvil

$E=\left\{r_{i} / 1 \leqslant i \leqslant n\right\}$ : etiqueta móvil

$\lambda=$ relación $u / u^{\prime}$

$q=$ parámetro del recuadro ampliado

$F\left(r, r^{\prime}\right)=$ función con valores binarios (criterio ocupacional)

$\omega=$ palabra (de ordenador)

$b, B=$ bits

$n_{b}=$ número de bits por palabra

$r_{x, u}=(x-u / 2, x+u / 2):$ recuadro» unidimensional

$p=$ penetración de dos intervalos

$s=$ separación de dos intervalos

$p_{\text {med }}=$ valor medio de $p$

$p_{\max }, p_{\min }=$ valores máximo y mínimo de $p$

$G(x), g(x)=$ función de distribución y función densidad de probabilidad de una variable aleatoria unidimensional

\section{résumé}

Dessin automatique de plans complexes, contrôle de positions occupées

J. Ortiz Herrera,
Dr. Ing. des Ponts et Chaussées

La conception à l'aide de computeurs de structures de bâtiment à un niveau d'automatisme pratiquement du cent pour cent exige la résolution de bon nombre de problèmes de plusieurs sortes. Parmi eux l'auteur choisit un sujet particulièrement intéressant, pour son applicabilité générique et pour pour son applicabile automatique des structures.

\section{summary}

Automatic Design of Complex Plans, Control of Occupied Positions

J. Ortiz Herrera, Dr. civil engineer

The computerized design of building structures at a level of an almost total automation give rise to a great number of problems of various kinds that have to be has chosen a topic that is of special interest because of its general field of from automatic dimensioning of structures.

\section{zusammenfassung}

Automatische Zeichnung Komplexer Plans, Kontrole Besetzter Stellungen

J. Ortiz Herrera,

Diefbau Ingenieur

Durch die fast völlig automatisierte Tragwerksberechnung entstehen etliche Probleme verschiedener Art, die gelöst werden mussen. Von diesen Problemen hat der Autor ein Thema gewählt, dass auf Grund
seines allgemeinen Anwendungsgebietes von seines allgemeinen Anwendungsgebietes von
besonderem interesse ist und weil es sich von den bereits schon klassischen Themen, die die automatische Dimensionierung von 